\title{
Los diarios de campo en la investigación social. El caso en el estudio "Socialización, aculturación y competencia intercultural. Un análisis empírico de familias multiculturales"
}

\author{
IGNACIA PEREA CRESPO \\ IUDESP. UNIVERSIDAD DE ALICANTE \\ BEGOÑ LÓPEZ MONSALVE \\ IUDESP. UNIVERSIDAD DE ALICANTE
}

\begin{abstract}
Resumen
El desarrollo de la investigación empírica en el ámbito de las ciencias sociales ofrece la oportunidad de tomar contacto con la realidad en más aspectos, incluso, de los que se prevén en un proyecto. En este texto se presenta, a modo de nota metodológica, algunas de esas llamadas de atención que el mismo desarrollo del trabajo de campo ha ido incorporando en cuanto a la siempre difícil labor de categorización social que, en algunos casos, hace cuestionarse las estandarizaciones sociológicas más básicas referidas a entornos en transformación.
\end{abstract}

Palabras Clave: trabajo de campo, cambio social, parejas mixtas, familias, migrantes

\begin{abstract}
The empirical research in social sciences offers the opportunity to take contact with the reality across the fieldwork in aspects which some times aren't considered in the beginning. This article presents some of the methodological notes generated during the fieldwork, which inform about the changing societies and the difficulty with some standard social classification.
\end{abstract} Keywords: fieldwork, social transformation, mixed couples, families, migrants 


\section{Introducción}

En las siguientes páginas se realiza una aproximación a la investigación "Socialización, aculturación y competencia intercultural. Un análisis empírico de familias multiculturales" (financiada por Ministerio de Educación y Ciencia; SEJ200505034/SOCI) en referencia a sus contenidos metodológicos y sus implicaciones en el desarrollo del trabajo de campo.

El tema central a tratar es el de los diarios de campo y cómo sus contenidos aportan información significativa a la investigación más allá de los datos recogidos; al contextualizar no sólo la información recopilada, sino también todo el proceso de recabación de datos con las anotaciones sobre las presencias, ausencias y, en especial, los procesos de búsqueda de observaciones.

En este caso, entendemos que estas anotaciones y reflexiones del trabajo de campo contenidas en los diarios, son necesarias dada la naturaleza metodológica arriesgada de una investigación que, como la planteada, no partía de la accesibilidad previa de los casos de estudio. La investigación responde a una lógica inventiva a partir de la cual conseguir reformular las perspectivas en el estudio de la temática sobre interculturalidad, razón por la cual, a través del diseño metodológico formulado, el desarrollo del trabajo de campo asume los riesgos de desarrollo de esta perspectiva, incorporando como instrumento de recogida extra de información los diarios de campo.

En primer lugar, la lectura de esta información complementaria posibilita retroalimentar el proceso de investigación incorporando toda los datos contenidos en los diarios de campo con respecto a la localización de casos de estudio, concertación de entrevistas y desarrollo de las mismas. En última instancia, con ello se nos ofrece la oportunidad de hacer confluir lo que podríamos denominar como teoría y práctica, completando, de esta manera, el círculo o espiral que conforma el proceso de investigación social.

\section{La muestra}

La investigación "Socialización, aculturación y competencia intercultural. Un análisis empirico de familias multiculturales" aborda la temática de la interculturalidad a partir del estudio de familias 
multiculturales. Para ello, el diseño muestral de partida elabora una muestra de familias mixtas donde la heterogeneidad es introducida sistemáticamente a partir de la combinación de los siguientes factores: nacionalidad de origen del miembro no nacido en España, género de la persona nacida en un país diferente de España, estatus sociolaboral comparativo intra-pareja y la presencia de hijos en el conjunto familiar (figura 1).

Figura 1. Combinatoria de factores de heterogeneidad muestral

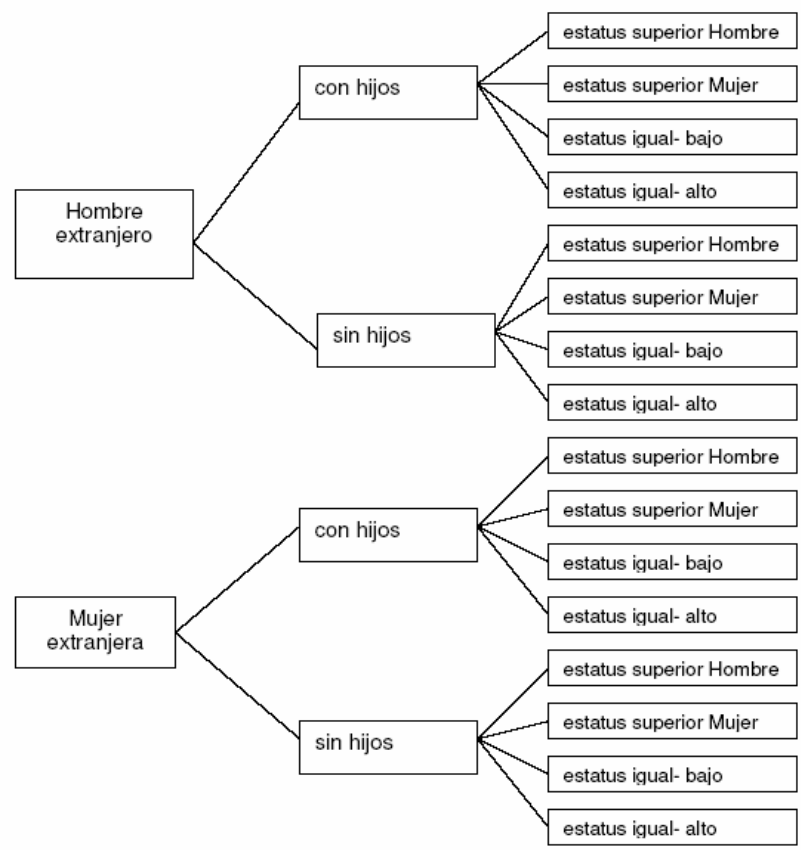

El esquema anterior se aplica a cada una de las nacionalidades seleccionadas en función de su mayoritaria presencia en el territorio (inglesa, alemana, ecuatoriana y marroquí), obteniendo una muestra compuesta por un total de 64 familias multiculturales; además del grupo de control formado por familias en las cuales ambos miembros de la pareja tengan como nacionalidad de origen la española (ocho familias con igual variedad en los factores de estatus sociolaboral e hijos que el resto de grupos).

El resultado de la combinatoria de tales factores es el de una muestra de gran heterogeneidad que permite alcanzar un amplio abanico de casos referidos a situaciones donde la interculturalidad, 
elemento en común de todas las familias, pueda ser filtrada a través de otras variables relevantes en el estudio de las relaciones interpersonales.

Desde este planteamiento de inicio arranca la labor de la recolección de datos con una gran exigencia en cuanto a la sistematización de éste; es decir, el proyecto planteado supone un estudio arriesgado en cuanto a su puesta en práctica en la fase de recolección de datos primarios debido tanto a los casos en sí (unidades multipersonales como son las familias), como a las exigencias de heterogeneidad definidas en el diseño.

\section{La localización de casos}

En primer lugar la localización de los casos correspondientes a familias multiculturales formados por las nacionalidades seleccionadas no cuenta con antecedentes en los canales usuales de contactación. Al tratarse de un estudio en el que hay implicación de personas extranjeras, las asociaciones, de diferente naturaleza, compuestas por residentes no españoles o que ofrecen algún tipo de servicio a inmigrantes y extranjeros fueron las primeras consultadas. No obstante, no resultaron ser una fuente importante de casos, lo cual ya arrojaba indicios de una lógica diferenciada de participación social entre población extranjera en general y aquélla que forma familia junto a una pareja española, como lo son los sujetos de nuestra búsqueda. Así, las organizaciones de inmigrantes de carácter socioasistencial que agrupan y/o atienden a población procedente de países incluidos en nuestra muestra, como son Ecuador y Marruecos, no resultaron ser fuentes importantes de casos de familias mixtas. Asimismo otras asociaciones de carácter cultural formadas en torno a la población procedente de Alemania o Gran Bretaña, tampoco resultaron fructíferas para nuestra búsqueda.

En general, se trata de canales a través de los cuales podemos dirigirnos hacia casos de extranjeros, pero no es así para los casos buscados en los que éstos hayan formado un proyecto conyugal junto a una pareja española. Este hecho, la carencia primera de resultados, ofrece los primeros indicios del comportamiento diferenciado, en cuanto a asociacionismo se refiere, de los miembros extranjeros que forman parte de familias mixtas. El desempeño de las funciones asociadas a la etapa de trabajo de campo permite, asimismo, reinterpretar tales indicios procedentes de la localización de los casos a 
la luz de las primeras informaciones recogidas, de manera tal, que las hipótesis referidas han sido inicialmente avaladas por el contenido de las entrevistas; apuntando uno de los focos analíticos a tener en cuenta: la conexión del miembro de la familia de nacionalidad no española con asociaciones y organizaciones relacionadas con su país de origen, así como la relación en general con población procedente de su mismo país.

Así lo indica también, aunque de un modo más matizado, los resultados de otra de las fuentes de localización empleada: la bola de nieve no ofrece resultados suficientes puesto que los miembros entrevistados de familias multiculturales no contaban necesariamente con contactos con otras familias de este tipo.

Por todo lo enunciado, la forma de localización de casos, dada la infertilidad de las estrategias anteriores, ha sido principalmente a través de los contactos personales y búsqueda activa en los entornos próximos de los mismos miembros del equipo investigador $\mathrm{y}$ colaboradores del estudio. Asimismo, se ha investigado sobre los nuevos canales de contactación a través de la red, sea a través de foros temáticos o dirigiéndonos a correos electrónicos oficiales para invitar a la participación del estudio.

En un marco general, el factor definitorio subyacente en estos problemas de localización de casos consiste en que la distribución de estas familias no es homogénea, las relaciones entre ellas no tienen porqué darse y sus intereses y estrategias de integración varían respecto a los núcleos tradicionales de localización de extranjeros de su misma nacionalidad, por lo cual tampoco son frecuentes en asociaciones definidas por la nacionalidad de origen.

Si observamos la escasez de fuentes potentes de casos como un elemento revelador a tener en cuenta en el análisis, aún lo es más atendiendo a éstas por nacionalidades. En cuanto a nacionalidades, las familias mixtas en las que uno de los cónyuges es originario de Marruecos han supuesto, sin duda alguna, mayores dificultades de localización (también de entrevistar como se detallará más adelante). Como explicación a este hecho, desde la labor de campo sólo pueden apuntarse algunas anotaciones y percepciones al respecto.

En general se ha observado un recelo por parte de los extranjeros extracomunitarios al plantearles el objeto de estudio, recelo 
que puede explicar en parte la carencia de casos y que pudiera ser que afectara, de alguna manera, más directamente a las familias españolamarroquís. Si bien desde la concepción de la investigación, hasta el diseño de la muestra, los factores impulsores han sido, obviamente, científicos, el estudio de familias mixtas que cuentan con un miembro extranjero de nacionalidad extracomunitaria ha de asumir ciertas suspicacias por parte de los sujetos del estudio; en especial cuando existe alguna coincidencia temporal con noticias difundidas a través de los medios sobre la celebración de matrimonios de conveniencia como estrategia de regularización de inmigrantes como fue el caso (apuntando específicamente a la población magrebí).

Pero sin incidir en una noticia en concreto, lo cierto es que a través de los medios de comunicación el entorno social está salpicado por informaciones de esta naturaleza, lo cual sumado a los estereotipos sociales entorno a la población inmigrante en general, apuntan algunos posibles en la explicación de los obstáculos del trabajo de campo desarrollado en este estudio en particular.

\section{La participación en el estudio}

La unidad de análisis de nuestro estudio es, como ya se ha mencionado, la familia; siendo la entrevista semiestructurada el instrumento de recogida de información. El hecho de que sea la familia la unidad de análisis supone que se ha de llegar a efectuar entrevistas a los dos miembros de la pareja (e hijo/a si es el caso), es decir, que no sólo se ha de contar con la disponibilidad e interés de uno de los miembros de la familia a colaborar en el estudio, si no con todos los integrantes de la misma.

Obviamente, para la organización del trabajo de campo se ha contado con este hecho y sus posibles obstáculos asociados. Teniendo en cuenta los posibles impedimentos para la concertación de las entrevistas, se ha desarrollado diferentes estrategias de facilitación previas, las cuales han ido alimentándose de otras establecidas a partir del desarrollo del proceso de recopilación de información.

Con el objetivo de minimizar el rechazo inicial a la entrevista individualizada realizada para cada miembro de la familia (dos o tres según los casos) y la posibilidad de que tal rechazo se manifieste no sólo en el momento de la contactación inicial, si no también una vez 
desarrollada la entrevista a la primera persona, se ha desarrollado diferentes estrategias de contactación con el fin de obtener un mayor grado de participación y colaboración. Así, se ha intentado contactar en primer lugar con la mujer, grupo inicialmente más dispuesto a la colaboración, se ha potenciado que el primer contacto con la familia fuera realizado por un conocido de ésta, etc. Estas estrategias de facilitación han permitido alcanzar un aceptable número de casos y se han acompañado por otras estrategias encaminadas a paliar el efecto de abandono (de otros miembros de la familia) y el desinterés que pudiera darse en los miembros a los que se ha llegado indirectamente y que pudiera repercutir en la calidad de los resultados obtenidos a través de las entrevistas personales.

No obstante, hemos de señalar que existen casos de abandono, es decir, familias en las que sólo se ha podido contar con la colaboración de uno de sus miembros y no se ha alcanzado a los otros miembros a entrevistar pero, en este caso, también las ausencias suponen información para el estudio dado que todos esos casos pertenecen al grupo de familias mixtas española-ecuatorianas y española-marroquís; de nuevo se trata de familias mixtas en las cuales uno de los miembros procede de un país extracomunitario.

Si bien las entrevistas estaban destinadas a todos los miembros de la familia, estos casos en los cuales no se ha podido llegar a todos sus miembros no son, de ninguna manera, desechables. El valor de la información está sujeto al contenido de la entrevista y ésta es desarrollada desde el punto de vista biográfico ahondando en la relación de pareja y los hijos si se tienen, así se produce en la entrevista un efecto espejo, es decir, a través de una sola entrevista, se llega a obtener información del resto de componentes de la familia.

\section{Las entrevistas}

En el estudio se realiza entrevistas personales semiestructuradas con un enfoque biográfico, profundizando en los aspectos relativos a la pareja y la familia. En general, podemos señalar tres bloques temáticos en las entrevistas: una primera parte relativa a los orígenes del/a entrevistado/a, un segundo bloque relativo a la relación de pareja e hijos, si es el caso, y un último bloque en el cual se ofrece la oportunidad de opinar acerca de diferentes temas de actualidad. 
En consonancia con los objetivos del estudio, se hace hincapié en la relación de pareja desde el momento en que se conocen, y las consecuentes relaciones con miembros de la familia extensiva, hasta la actualidad. Se trata de un punto central en la entrevista que, en ocasiones, se encuentra con la intención por parte de la persona entrevistada de soslayar tales cuestiones (percibida por parte del equipo entrevistador). Sin contar con los inconvenientes de la entrevista en profundidad cara a cara ya conocidos, el trabajo de campo recoge que en esta ocasión también tiene un peso relevante otras cuestiones relacionadas con el hecho de que sea la familia la unidad de análisis.

La deseabilidad social afecta de forma distintiva en los temas relacionados con la familia, asentándose en la extendida aunque difícilmente confesable norma que dicta aquello que podría enunciarse como que lo que ocurre en la familia se queda en la familia. Las impresiones extraídas en el desarrollo de las entrevistas cuentan la sombra de dicha norma en una entrevista en la que se aborda relaciones y opiniones referidas a la familia de origen, la familia política y la propia unidad familiar, además de ser realizada a más de un miembro de la misma familia.

No obstante, las impresiones recogidas por el equipo entrevistador son positivas en cuanto a la honestidad de los participantes en el transcurso de las entrevistas, si bien también se indica cierta propensión, en algunos casos, a soslayar las cuestiones más personales relacionadas con familia extensiva. Ello es debido, no sólo al carácter personal de los temas, si no también a la naturaleza distintiva de los estudios enfocados a la familia en cuanto a trabajo de campo se refiere.

\section{El diseño y la realidad social}

Los estudios sociales empíricos, en especial los que indagan sobre cuestiones sociales emergentes, toman el pulso de una realidad social caracterizada por su naturaleza cambiante. De ahí que en algunos casos el recopilatorio de casos de difícil clasificación, las incidencias en la labor de campo en cuanto a la misma concepción de los casos, y otras anotaciones tomadas durante el desarrollo de recolección de datos, resulta ser un campo fértil en la producción de observaciones para la reflexión y la apertura de nuevas líneas de investigación. 
Así ha ocurrido en nuestro estudio en el cual los factores de variabilidad empleados en el diseño de la muestra han debido observarse para cada uno de los casos poniendo de manifiesto la complejidad de la realidad social; verificando el planteamiento de que no resultan tan obvias ni aún las clasificaciones más básicas.

Algunas de las incertidumbres son ya clásicas en el estudio sociológico, como es el caso del establecimiento del estatus sociolaboral de los miembros de la pareja. Se ha contado con un indicador combinado de nivel educativo y empleo desempeñado que, además de contar con las limitaciones de cualquier indicador creado para la medición del estatus, contiene las particularidades de la población mixta, esto es, en ocasiones no existe equivalencia de los mismos indicadores objetivos para población autóctona y foránea. Ejemplificando este punto, podemos señalar una de las cuestiones que hemos debido valorar para cada uno de los casos, como es la relatividad del nivel educativo, en especial asociado a su proyección laboral, para los casos de estudios no homologados en el país de residencia (como es el caso de muchos extranjeros en España). Para estos casos se ha debido tener en cuenta que el estatus social se resiente en aquellos casos en los cuales el nivel de estudios citado no está acorde con el puesto de trabajo por imposibilidades legales (dificultades de homologación), es decir, cuando el nivel educativo entendido como medio de posicionamiento en la jerarquía laboral pierde validez.

Asimismo, las cuestiones definitorias de familia tanto en la relación de la pareja, como en la pertinencia de considerar la tenencia de hijos, han mostrado una enorme variabilidad interna indicativa de las múltiples tipos de familia en unos casos, y la multitud de relaciones sociológicamente relevantes entre sus miembros en otros. El trabajo empírico nos confirma la presencia de un gran abanico de posibilidades: parejas no vinculada legalmente que conviven, parejas casadas que no conviven la mayor parte del tiempo o parejas casadas pero que conviven con una pareja diferente a su marido o esposa; con hijos comunes de los cónyuges, de ambos pero no comunes, comunes a sólo uno de los miembros o de todo tipo; algunos hijos viven en el domicilio, otros no han convivido nunca con la pareja actual, algunos ni siquiera les conocen, etc. En definitiva, asistimos a cómo las historias de vida entrelazadas de una sociedad que aumenta las 
posibilidades de relación y convivencia salen a la luz en el mismo momento en que se toma contacto con ella a través de la investigación empírica.

Todo ello ha hecho que se tome (y retome) conciencia de la realidad social, se asuma que ningún diseño puede abarcarla en toda su complejidad y se estudie cada uno de los casos en concreto asumiendo las enseñanzas aportadas por cada uno de ellos.

No obstante, ha quedado al margen casos que hubieran sido interesantes estudiar pero que desbordaban los límites de la investigación planteada. Ese es el caso de las familias monoparentales de origen intercultural, así como el de las parejas mixtas homosexuales, formalmente establecidas o en convivencia, que hubieran podido aportar observaciones acerca del género y la interculturalidad realmente valiosas. Si bien estos casos, no han podido englobarse en nuestra investigación, a pesar de la adaptabilidad de éste impulsada por el desarrollo del trabajo de campo, resultan valiosos para la apertura de nuevas perspectivas de estudio. Desde este enfoque, se valida la pertinencia de recopilar las observaciones, dudas y anécdotas de todas las fases del trabajo de campo y se señala cómo toda esta información resulta útil para señalar nuevas líneas de investigación; ideas surgidas desde una posición privilegiada de acercamiento-distanciamiento de la realidad social como son las posiciones asociadas al desempeño del trabajo de campo.

\section{Conclusiones}

En este artículo se ha expuesto algunas de las incidencias y observaciones recogidas durante el desarrollo de la fase de campo en el estudio "Socialización, aculturación y competencia intercultural. Un análisis empírico de familias multiculturales", ejemplificando el valor de los contendidos de los diarios de campo en la investigación empírica, más allá del tratamiento accesorio que suelen tener en el conjunto de los elementos de la investigación. Se trata de una inclusión de gran utilidad cuando se lleva a cabo una elaboración conjunta de los contenidos de la información contextual e incidencias de campo en relación a los objetivos del estudio, tal y como se ha expuesto en las páginas precedentes. 
Además, como ya hemos señalado al inicio, la investigación plantea un diseño arriesgado que se ha reflejado en el transcurso del trabajo de campo, lo cual conlleva una gran cantidad de incidencias, pero también de retos metodológicos, ofreciendo la oportunidad de desarrollar un trabajo de retroalimentación en las fases de la investigación.

Es esto último lo que impulsa la redacción de estas páginas en las cuales se describe algunas incidencias ocurridas en el trabajo de campo, así como la asociación de las observaciones de una ingente labor de campo con el tema central de estudio y, consecuentemente, las hipótesis que manan directamente del trabajo empírico y que pueden ser de utilidad no sólo a la labor analítica posterior, sino también a la investigación futura. Todo ello posible gracias a un diseño arriesgado que no sólo trae consigo dificultades de ejecución, sino que además plantea retos interesantes tanto a nivel estratégico, como analítico; ofreciendo la oportunidad de acercarnos con humildad y honestidad a la máxima por todos conocida de que la realidad social es compleja.

Los datos aquí aportados validan la necesidad de contextualizar la información obtenida por medio de la entrevista, demuestra la pertinencia de integrar información primaria de la persona entrevistada y la entrevistadora al proceso de investigación y, en suma, cierra el círculo de la investigación social preparando los datos optimizados para el análisis final. Por último, el proceso de retroalimentación introducido por el empleo de los diarios de campo, proporciona nuevos elementos de reflexión para nuevas líneas de investigación futura. 